\title{
Design of Intelligent Irrigation System for Greenhouses Based on Internet of Things
}

\author{
Hong-Jun GUa, Ji LI, Shi-Jun LI ${ }^{b}$, He GONG, Jian LI, Li-Xin HOU \\ Jilin Agricultural University, Changchun 130118, Jilin, China \\ aghj_dennis@163.com, 'Isj0883@sina.com
}

Keywords: IOT, pump controller, soil moisture sensor, intelligent irrigation.

\begin{abstract}
This paper designs a new irrigation system for greenhouse based on Internet of things (IOT) which realizes intelligent control to the water pump. This system consists of ZigBee module, soil moisture sensor, controlled circuit, remote control unit, mobile, computer, AC contactor, pump, display circuit and keyboard. ZigBee module compares the data from soil moisture sensor with preset value from the mobile, computer or keyboard to control the pump automatically. Users can also operate it manually or remotely to change the pump working state. The system realizes the intelligent control for irrigation system of the greenhouse. After a long work verification, the system has the performances of safety and reliability, low cost and can be embedded into agricultural IOT.
\end{abstract}

\section{Introduction}

The greenhouses with the functions of automatic detection and control can provide a good growth environment for crops. In recent years, with the improvement of people's living standard, greenhouses are increasing rapidly [1]. How to reduce operating costs, improve efficiency, save labor, and realize accurate control of environmental parameters has been a research topic in the current agricultural field [2].

In traditional agriculture, farmers can only rely on intuition as to the crop irrigation. With the development of science and technology, modern agriculture is developing towards the intelligent direction [3]. Agricultural IOT can solve the problems, such as whether fruits and vegetables needs watering, fertilizing, spray insecticide, and how to ensure the temperature, moisture, light, $\mathrm{CO}_{2}$ concentration of the greenhouse [4]. Modern intelligent monitoring and control system can improve the growth environment for the crops [5]. Of course, farmers can also manually operate the system via mobiles, computers, buttons and keyboard.

Water pumps are playing an important role in the agricultural fields. In the greenhouses, introducing the new technology and advanced equipment is beneficial to improve the irrigation efficiency, save water and labor[6]. This paper presents a new irrigation system which can intelligently send the useful related data to the users and accept the control commands.

\section{Overall System Framework}

The intelligent control system uses ZigBee module as the core, which can communicate useful information with mobiles and computers, and send the commands to the controlled circuit of the pump. The remote controller can realize the near distance wireless control of the pump. At the same time, manual switches can also change the working state of the pump. The overall system framework is shown in Fig. 1.

ZigBee module acquires the data from the soil moisture sensor and sends them to the computer with Internet access via the gateway. The computer sends the data to intelligent mobile. The administrators can inquire the soil moisture data and send watering command to the pump via the mobile or the computer. The computer can analyze the gathered information and prepare for agricultural big data. The farmers can modify the preset soil moisture via the mobile, the computer or the keyboard. The displaying screen can show the current time and date, preset moisture value and collecting moisture values of many nodes. Several important parts are introduced in the following. 


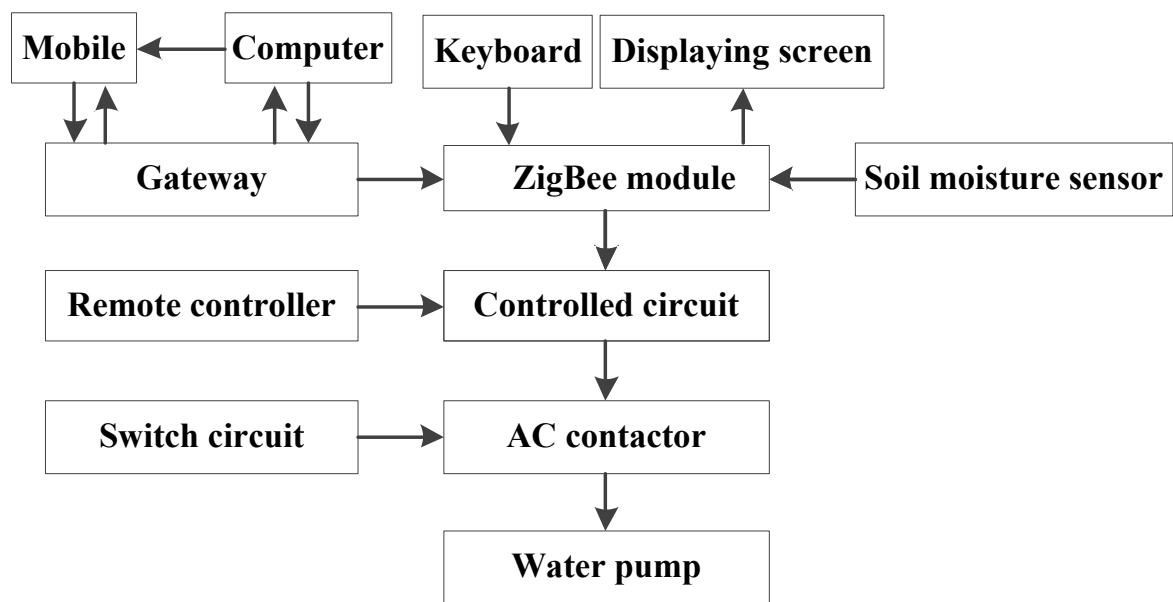

Fig. 1 The overall system framework

\section{ZigBee Minimum System}

The CC2530 is chosen as the control core. It is a true system-on-chip solution for IEEE 802.15.4, ZigBee and RF4CE applications. The CC2530 combines the excellent performance of a leading RF transceiver with an industry-standard enhanced $8051 \mathrm{MCU}$, in-system programmable flash memory, 8-KB RAM, and many other powerful features. This system adopts CC2530F256 with $256 \mathrm{~KB}$ of flash memory. The detailed minimum system diagram is shown in Fig .2.

This chip can collect the moisture data, compare it with the preset value and decide whether watering task should be started. ZigBee has ad-hoc network function, so the moisture data can be sent between nodes, and at last the data can be accepted by the computer and mobile with the help of gateway.

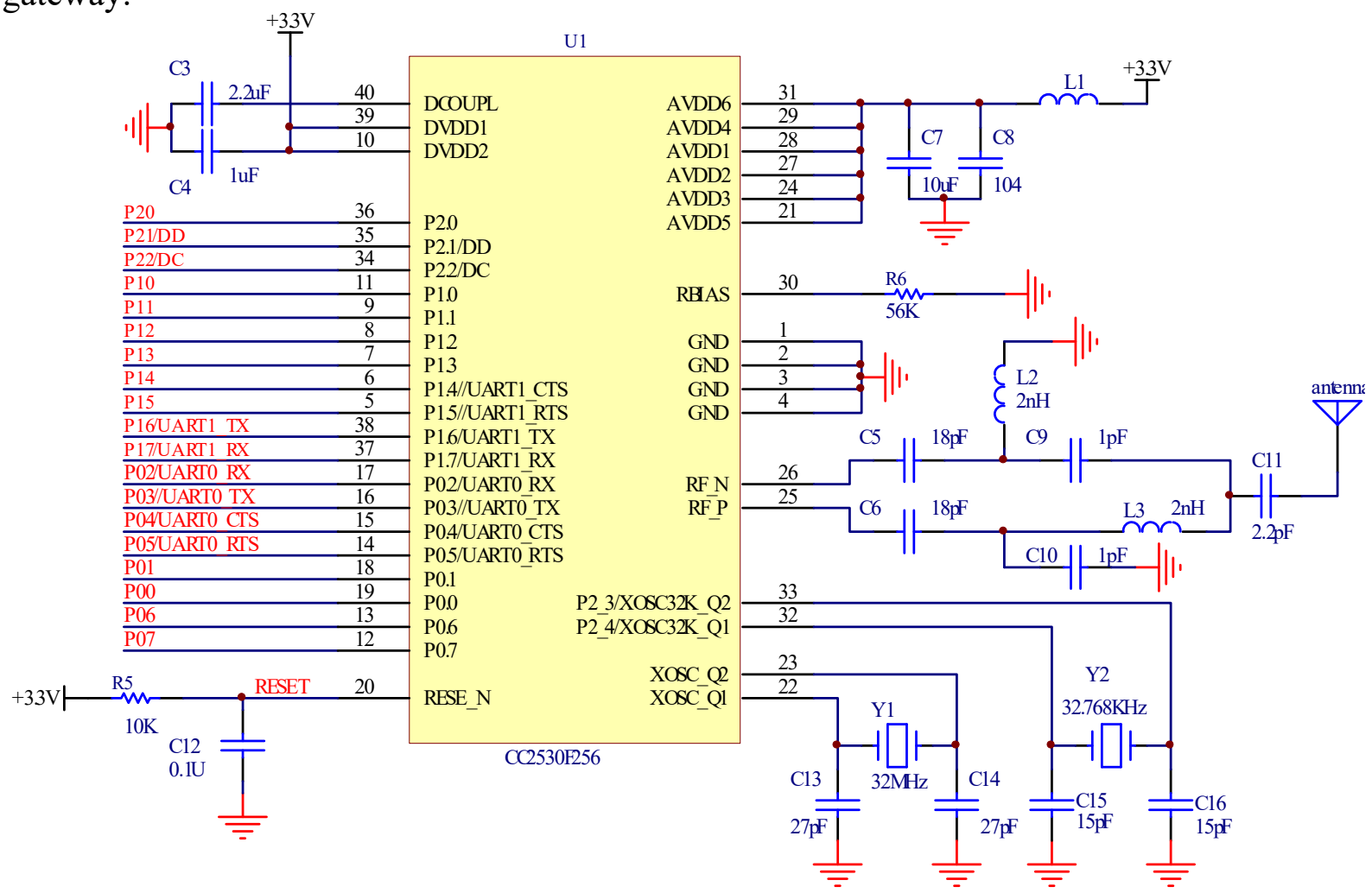

Fig. 2 CC2530 schematic diagram

\section{Remote Control Unit}

Remote control unit uses PT2262/HS2272 to design the wireless transmitting and receiving modules, respectively, and the products are shown in Fig. 3. The communication distance of the 
device with the operating voltage range $3-12 \mathrm{~V}$ can reach 1000 meters, which can satisfy the application in the greenhouse.

The transmitter has 2 keys representing for the commands 'on/off'. The receiver gains the corresponding information to operate the pump. The users can control the pump at the entrance of the greenhouse.
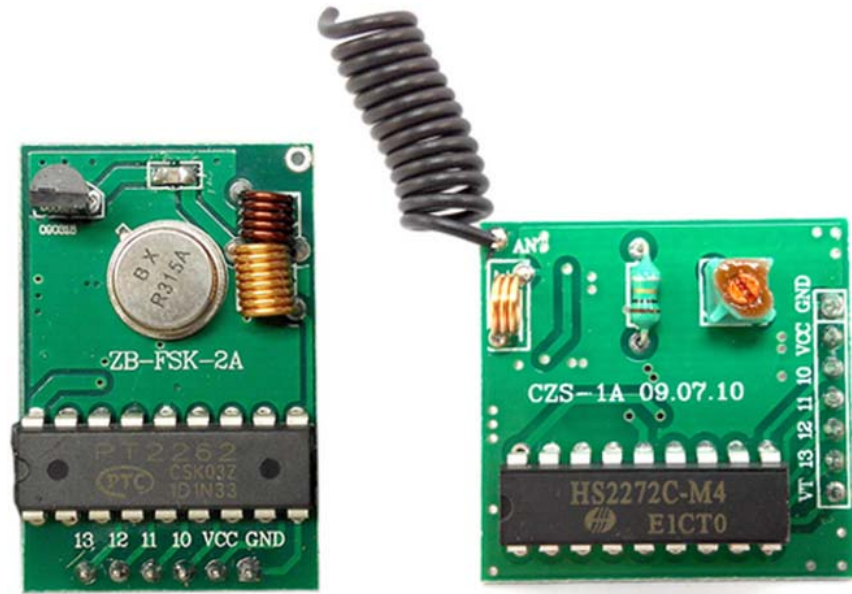

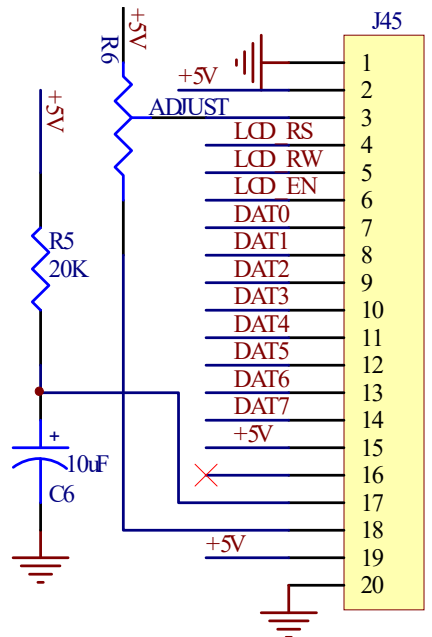

Fig. 4 Displaying circuit schematic diagram

Fig. 3 Products of remote control unit

\section{Displaying Circuit Design}

Displaying circuit adopts LCD12864 which can show the current time and date, moisture values of many nodes and the state of pump. As shown in Fig. 4, LCD12864 with 20 pins communicates with CC2530 by DAT0 - DAT7, LCD RS, LCD RW, and LCD EN. DAT0-DAT7 means transmitting data or commands. LCD_RS decides DAT0-DAT7 representing data or commands. LCD_RW means 'read' or ,'write' operation. LCD_EN gets a pulse, and data or commands have been written in the LCD12864.

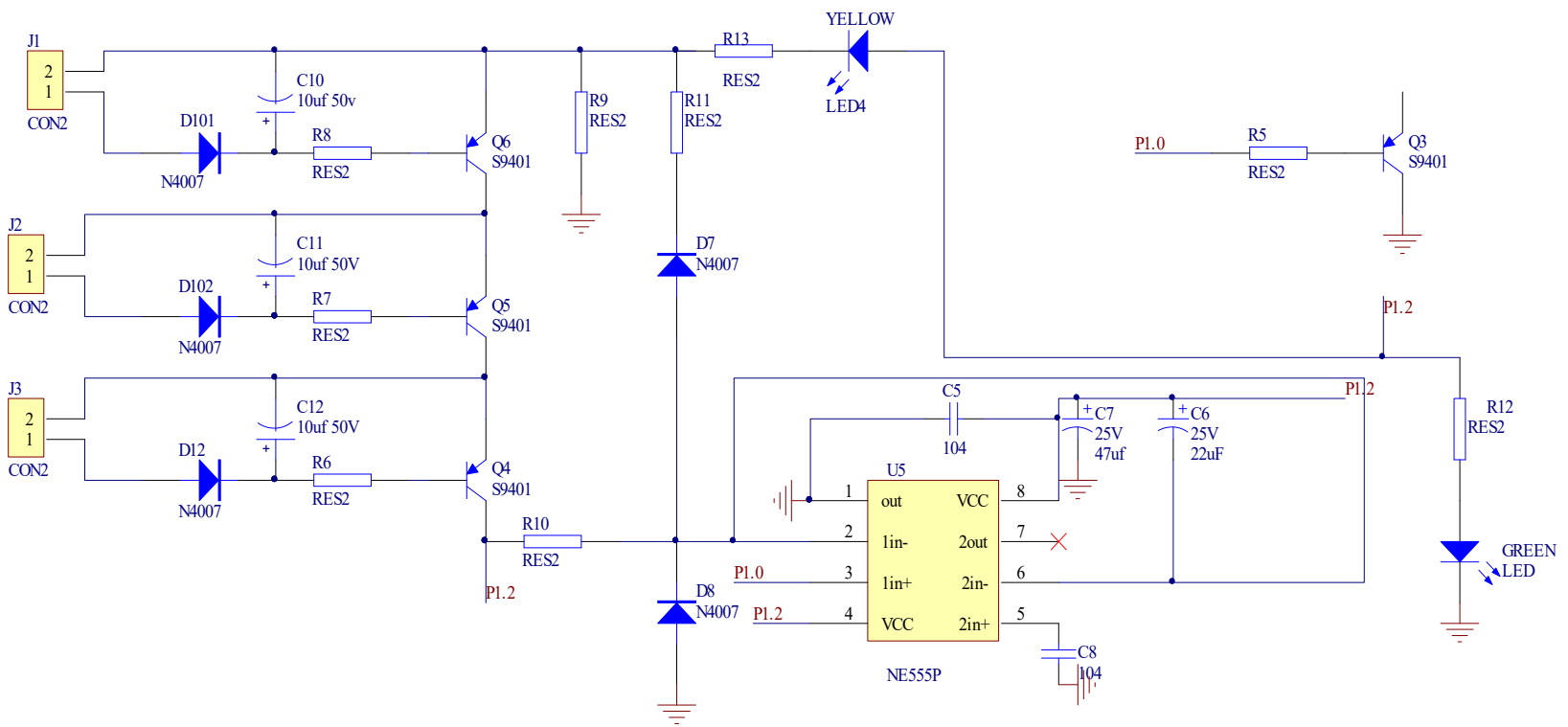

Fig. 5 Phase lack protection circuit

\section{Phase Lack Protection Circuit}

In the controlled circuit, phase lack protection circuit can prevent the motor of the pump from destroying while three-phase motor lies in the missing phase operation. The following shows the basic principle of the circuit. 
$\mathrm{J} 1, \mathrm{~J} 2, \mathrm{~J} 3$ represent three inductance coil set in two phase, respectively. When three-phase power lines are in good condition without self-induced electromotive force in three inductance coil, and P1.0 pin has low level. So the transistor Q3 lies in the conduction state, relay circuit work normally, and the AC contactor controlled by the relay circuit works well. When one of the three phase coils has no current, inductive electromotive force is produced between in the induction coil $\mathrm{J} 1, \mathrm{~J} 2$ and $\mathrm{J} 3$, then the triodes Q4 - Q6 lie in the off state. The third pin P1.0 of the timer 555 outputs the high level which lets Q3 lie in the off state. So the relay works to shut off the main circuit power supply, and the motor can be protected.

\section{Conclusions}

This paper designs a new irrigation system for greenhouse based on IOT. This system made up of many modules uses CC2530 as the core. CC2530 compares the data from soil moisture sensor with preset value from the mobile, computer or keyboard to control the pump automatically. Pump state is controlled by manually, remotely, and automatically. In this paper, several important parts,including design schematic diagram and produces, have been shown. This system can save a lot of labors and let fruits and vegetables watered timely. After a long work verification, the system has the performances of safety and reliability, low cost and can be embedded into agricultural IOT.

\section{Acknowledgments}

The authors wish to express their gratitude to the projects: Design of Standardized Breeding System for Rabbits Based on Internet of Things from Education Department of Jilin Province, Jilin Province Economic Structural Adjustment Leading Fund Special Project (No. 2014Y108) and Changchun City Science and Technology Plan Project (No. 14nk029, No. 13KG71), Key Tackling Item of Jilin Province Science \& Technology Department (No. 20140204045NY), Jilin Province Science \& Technology Department Project (No. 20150204058NY, No. 20140204045NY) for their generous support of this work.

\section{References}

[1] WANG Hui, WANG Sheng-Wei, and WANG Zhi-Qiang, et al. The Mobile Intelligent Control System of Greenhouse Based on IoT and WiFi [J]. Beijing: China Agricultural University, 2014, (4), in Chinese.

[2] WANG Zhe. Design of Intelligent Greenhouse Based on IOT and Solar Cells [D]. Qiqihar: Qiqihaer University, 2015, in Chinese.

[3] LIN Hong. Reserch on Intelligent Greenhouse Environmental Monitoring System [J]. Baoding: Hebei Agricultural University, 2014, in Chinese.

[4] CUI Wen-Shun, YUAN Li-Zhe, and CUI Shuo, et al. Design and Implementation of Sunlight Greenhouse Service Platform Based on IOT and Cloud Computing [C]. International Conference on Measurement Information and Control, 2013: 141-144, in English.

[5] Boughanmi, H, Lazaar, M, and Bouadila S, et al. Thermal Performance of a Conic Basket Heat Exchanger Coupled to a Geothermal Heat Pump For Greenhouse Cooling under Tunisian Climate [J]. Energy and Buildings, 2015: 87-96, in English.

[6] GU Hong-Jun, ZHU Shi-Dong, and YANG Yan-Fen, et al. Design of Intelligent System for Watering Flowers Based on IOT [C]. Advances in Engineering Research, 2015: 1469-1473, in English. 